\title{
Translational cancer research towards Thailand 4.0
}

\author{
Kanlayanee Sawanyawisuth $^{\mathrm{a}}$, Chaisiri Wongkham ${ }^{\mathrm{a}}$, Chawalit Pairojkul ${ }^{\mathrm{b}}$, Sopit Wongkham ${ }^{\mathrm{a}, \mathrm{c}, *}$ \\ a Department of Biochemistry, Faculty of Medicine, Khon Kaen University, Khon Kaen 40002 Thailand \\ b Department of Pathology, Faculty of Medicine, Khon Kaen University, Khon Kaen 40002 Thailand \\ c Center for Translational Medicine, Faculty of Medicine, Khon Kaen University, \\ Khon Kaen 40002 Thailand
}

*Corresponding author, e-mail: sopit@kku.ac.th

\begin{abstract}
Cancer is still a major cause of mortality in the Thai population. The heterogeneity and complexity of cancer make this disease often result in ineffective treatment and a fatal outcome. Primary prevention for a knownrisk cancer is one way to minimize the number of new cancer cases; however, effective treatment with reasonable cost-effectiveness is important for cancer patients. Basic and preclinical cancer research are necessary for better and deeper understanding of the nature of cancer. Several research initiatives should be implemented to translate the preclinical developments to clinical outcome. High specificity and sensitivity of tumour markers in serum or secretory fluids are helpful for diagnosis of cancer before advanced, high cost, or invasive diagnoses are implemented. A set of potential markers instead of a single marker should be researched to increase the diagnostic power of the tests for a particular cancer. Simplicity, low cost, and highly effective diagnostic power of the test is also required to validate a test for clinical use. The knowledge generated at the molecular level together with the advanced technologies of next-generation sequencing, cellular and molecular biology, and computational biology, provide a new trend of precise treatment against individual cancers. Histoculture drug response assays or in vitro chemo-sensitivity assays, genomic profiling of tumours by next-generation sequencing, drug repositioning for cancer, and chimeric antigen receptor T-cell therapy are exciting approaches that lead to precise and effective therapy for cancer patients. In this review, several new directions for the precise diagnosis and effective treatments of cancer are highlighted.
\end{abstract}

KEYWORDS: precision medicine, histoculture drug response assay, repurposing drugs, CAR T-cell

\section{INTRODUCTION}

Cancer is considered a leading cause of death worldwide in developed and developing countries, including Thailand. The number of cancer cases and deaths is expected to grow rapidly. As noted in GLOBOCAN 2012, approximately 8 million people died of cancer and 14 million new cases were recorded worldwide ${ }^{1}$. The most commonly diagnosed cancer in each country varied considerably. Based on GLOBOCAN 2012, prostate cancer was the most common cancer among males in North and South America, and Europe while the leading cancers among males varied substantially in Africa and $\mathrm{Asia}^{2}$. Breast cancer was the most common cancer among females in North America, Europe and Oceania, whereas breast and cervical cancers were the most frequently found cancers in Africa and most of Asia. According to the World Health Organization, the numbers of new cancer cases are expected to rise by about $70 \%$ over the next 20 years $^{3}$.

Cancer is also the highest cause of death of
Thais. The mortality rates of the Thai population in 2015, analysed from the Health Information System Development Office, Ministry of Public Health of Thailand (www.hiso.or.th) revealed that 417 per 100000 Thais died because of cancer, followed by kidney failure (100), accident (98) and diabetes mellitus (75) (Fig. 1). Among all cancer deaths, lung cancer was highest in the north, while liver cancer was extremely high in the north-east. Breast and cervical cancers seemed to be distributed over the country with lower rates in the north-east and the south (Fig. 2). Except for cervical cancer that seems to have been stable for a couple years, mortality rates of all major cancers in Thailand have an increasing trend. This may be due to the changes of lifestyle that increases exposure and accesses to risk factors, decreases physical activity, and changes eating habits and behaviour.

Thailand is now facing an ageing society. Low birth rates and longevity of the elderly population moves Thailand towards an ageing society. Of any developing country in East Asia and Pacific, Thailand together with China, has the highest share 

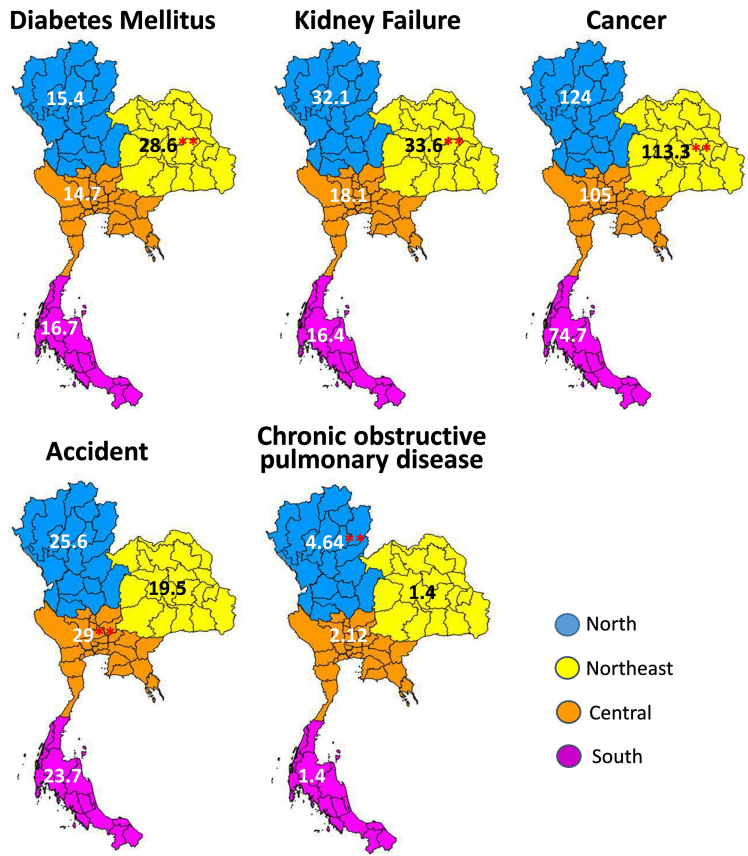

Fig. 1 The major causes of mortality in the Thai population, in 2015. The geographical distribution of mortality rates of the major causes of death in the Thai population in 2015. Cancer was the highest cause of death, followed by kidney failure and accidents. The numbers are rates of mortality per 100000 persons; ** indicates the highest mortality rate. Data from the Health Information System Development Office, Ministry of Public Health of Thailand (www.hiso.or.th).

of elderly people ${ }^{4}$. As the occurrence of cancer increases with age, it can be presumed that there will be a growing cancer incidence and mortality in the Thai population and the rising of healthcare expense for cancer patients in the next decade. Besides the primary prevention, effective diagnosis, treatments and care for cancer patients should be considered. The research and development to achieve an early and definite diagnosis as well as effective treatments for cancer patients should be intensively evaluated. This article is intended to share the perspective of cutting-edge biomedical research that may ultimately contribute to specific diagnosis and better treatments for cancer patients towards Thailand 4.0.

\section{INNOVATION OF TUMOUR MARKERS FOR DIAGNOSIS AND PROGNOSIS OF CANCER}

Cancer is a group of diseases that can occur in any tissue or organ with a common characteristicuncontrolled cell growth. Cancer cells divide uncon-
Mortality rate/100,000

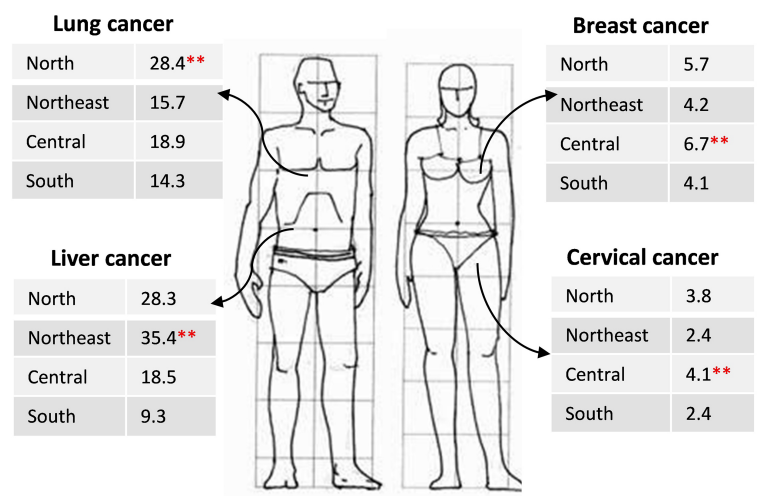

Fig. 2 Four major causes of cancer death in the Thai population in 2015. The leading cancers among Thai males in 2015 was liver cancer, followed by lung cancer, whereas those in Thai females was breast cancer and cervical cancer. The numbers indicate rates of mortality per 100000 persons and ** indicates the highest mortality rate. Modified figure from http://how2drawrunggg.blogspot.com/ 2014/01/humanscale-1-7-4-normal-7-dialistic-8.html.

trollably, form masses and in the advanced stage, metastasize to distant organs. Most fatal cancers are slow growing and have no specific symptoms at the beginning, thus almost all patients are diagnosed when cancers have metastasized. The potentially curative treatment is surgical resection, however, this can only be offered effectively for early stage cancers. Early detection of cancer is, thus crucial to reduce morbidity and mortality. Advanced imaging techniques such as X-rays, CT scans, MRI scans, PET scans, and ultrasound scans are used to detect tumour masses. These techniques, however, have limited sensitivity in detecting small masses or metastatic foci and are very much dependent on the experience of the operators. In addition, they are available only to a limited number of patients due to their high cost. Tumour markers then play a key role for primary diagnosis of suspected cancer in persons before using invasive or high cost diagnostic technologies.

Tumour associated markers are substances produced by a tumour itself or by the body as a response to the tumour. These biomarkers can be detected in tumour tissues and its microenvironments or body fluids, such as whole blood, serum, urine, and saliva. Several biomolecules reflecting the biological processes of cancer cells can be used as tumour markers. They can be proteins, glycoproteins, DNA, long non-coding RNA (lncRNAs), mi- 
croRNAs (miRNAs), metabolites, tumour associated macrophages and fibroblasts, circulating tumour cells and white blood cells. Tumour markers are of benefit not only for diagnosis, but also for prognosis and prediction of drug response. Some markers are good for screening as a health checkup. Long-term surveillance should be provided for a person who is positive for these markers. An early stage cancer, may then be diagnosed during follow up. Some tumour markers are of benefit for cancer diagnosis. These markers should be determined in combination with other investigations for definite diagnosis of a particular cancer. The prognostic markers are suitable to provide the information for clinicians to select the appropriate and effective treatment for patients. These markers mostly reflect the clinical manifestations of patients and are associated with patient survival or tumour staging. Some tumour markers are associated with drug responses or idiosyncratic genetic problems and can be used to predict the responsiveness of patients to the drug treatment.

The present high-throughput and large-scale analytical technologies, i.e., genomics, transcriptomics, proteomics and metabolomics, offer researchers an opportunity to discover a number of potential markers that could be used for diagnosis, prognosis or prediction of treatment responses for an individual patient. While many of them even hold a promising potential to use in clinical practice, many of them may not identify a particular type of cancer. As cancer is heterogeneous, it is well accepted that a combination markers may have better diagnostic power than a single marker. Using a ratio of two serum proteins may have high diagnostic and predictive powers in several cancer types. A comparison of serum proteomes from cholangiocarcinoma (CCA) patients and healthy controls, differential amounts of $\alpha_{1} \beta$-glycoprotein (A1BG) and afamin (AFM) were identified in sera from CCA patients ${ }^{5}$. Determination of these two serum proteins in a single blood test as an A1BG/AFM ratio could differentiate CCA patients with $84 \%$ sensitivity and $88 \%$ specificity. Besides, the albumin to $\gamma$-glutamyltransferase ratio ${ }^{6}$ and the aspartate aminotransferase to neutrophil ratio ${ }^{7}$ was proposed as a potential prognostic markers for CCA. A suitable combination of markers can give a precise diagnosis for a specific cancer type with satisfactory sensitivity and specificity for clinical implications. Most researchers selected the markers as a combination based on the physiological functions of those particular molecules. Machine learning decisions can effectively assist researchers in selecting a combination of potential markers with designed specificity and sensitivity. Using a decision tree diagram built by the C4.5 algorithm, 2 of the 8 diagnostic markers were selected to distinguish CCA patients from non-CCA subjects with sensitivity, specificity and accuracy $\geqslant 95 \%{ }^{8}$. With the high sensitivity, specificity and accuracy, a simple blood test could be the way to replace the biopsy; the gold standard for detecting cancer.

Based on a search on Pubmed from 2000-2017, there were 645 publications published by authors from Thailand on markers and cancers. Only a limited number of these, however, could be translated to clinical practice. To discover novel and high impact tumour markers available in clinical practice, collaboration and integration of knowledge among different disciplines are the keys to success. Biomolecular researchers, clinicians, biostatisticians, bio-informaticians, granting agencies and private sectors should join hands and set up a national project to innovate development of diagnostic tests for cancers that have a high incidence in Thailand. Evaluation of the usefulness of candidate tumour markers in routine pathological diagnosis is the next step needed to analyse their values. Systematic reviews and meta-analyses should be evaluated to clarify the impact of the potential markers for particular cancers and to prevent over-diagnoses. The detection techniques and the cohorts of studies with appropriate clinical data and precise sample collections are of importance and should be considered. A nationwide biobank to collect bio-samples taken with standard protocols should be set to support the discovery of novel tumour markers. In parallel, the non- or low invasive sample collection techniques, as well as the simple and affordable determination methods should be taken into account. Finally, towards the Thailand 4.0 policy, the possibility of the determination of the manufacture of tumour markers as commercially available test kits should be assessed.

\section{PRECISION MEDICINE: AN UPCOMING APPROACH FOR EFFECTIVE TREATMENT OF CANCER}

Many efforts and huge budgets have been invested to discover new drugs for cancer treatment. Low response rates and short overall survival, however, are still the norm. High recurrence rates and drug resistance are the main problems of ineffective treatments using either chemotherapy or targeted therapies. Furthermore, patients with similar cancer 
types frequently exhibit different drug responses to the same drug treatment. This observation has lately been shown to be the different molecular signatures of cancer in individuals that takes into account the diverse drug responses in individual patients. Not only the histopathology and clinical features of the cancer, but the specific molecular features of the cancer that drive and regulate the behaviour of cancer in a particular individual. Several studies have indicated different molecular signatures among individual persons with similar cancer types. For example, different expression patterns in transcriptomes, epigenomes and mutations were reported recently among cholangiocarcinoma patients from liver fluke and non-liver fluke associated cancers ${ }^{9-12}$. A "one-size-fits-all approach", in which the treatment strategies are developed for the average person, therefore, may not be not effective. To face this fact, an upcoming approach of 'precision medicine', in which the treatment is arranged according to individual variability in genes, environment, and lifestyle of each person, is recommended ${ }^{13}$. The cancers could be classified according to their expression profiles to predict the drug responsiveness of cancer in an individual as a good or poor response to the cancer. This is not only to increase the response rate and cost-effectiveness of the outcome but also avoids the opposite adverse effect of cytotoxic drugs for those who will not tolerate the treatment. This approach provides clinicians and researchers a means to predict more accurately the best treatment that will effectively work for individual cancer patient.

Precision medicine holds promise for improving treatment for cancer and many other diseases. Precision medicine is still young in Thailand. To establish this approach, physicians and medical scientists have to prepare many aspects in the drive for "precision medicine for cancer" in the country towards Thailand 4.0. Basic research is needed for a better understanding of biology, molecular signature sand underlying mechanisms of specific types of cancer in Thai patients. This information is of value not only for improving the treatment but also for diagnosis and prevention. As Thailand 4.0 targets a high value economy, basic research is necessary to analyse and filter the potential genes that may reflect the prognosis and drug response. Special cellular and molecular technologies, such as histoculture drug response assays, organoid and patient tissues derived xenograft models, RNA sequencing (RNAseq), and data mining are required.

\section{IN VITRO-GUIDED THERAPY IMPROVES RESPONSIVENESS TO DRUG THERAPY IN CANCER PATIENTS}

The histoculture drug response assay (HDRA) or in vitro chemo-sensitivity assay were developed in considering the urgent need for chemo-resistance prediction in cancer patients. Several clinical studies including colorectal, gastric and lung cancers revealed that inhibition rates obtained with HDRA can predict clinical responses to chemotherapy ${ }^{14,15}$. A study reported in Ref. 16, demonstrated that HDRA appeared to be useful to cancer patients for prediction of responses to chemotherapy. In this study, tumours from 329 various cancer patients who underwent chemotherapy were cultured and tested with different chemo-drugs for 5 days. The overall survivals were compared between patients with HDRA guided therapy and those with empiric therapy. The results indicated that the overall survivals in patients treated with HDRA guided therapy were significantly prolonged compared to those with empiric therapy. Similar results were obtained in a report of 359 lung cancer patients studied ${ }^{17}$. Using HDRA to evaluate the chemo-sensitivities of lung tumour tissues to several chemotherapeutic drugs, patients who received HDRA guided therapy are reported. They found that the evaluability and predictability of HDRA were high and could be used to predict clinical responses. Several studies demonstrated the strong association of in vitro and in vivo effects ${ }^{17}$, however, it is hard to exclude selection bias and confounding as an influence on the results. Future research should be conducted with well-designed randomized trials to measure survival outcomes.

HDRA is a representative of an in vitro drug response test for anticancer agents in individuals before starting the chemotherapy. It provides a reliable drug sensitivity test with a simple and low cost method. The advantages of the HDRA over other single cells suspension methods may be due to good cell viability and a more natural patient environment. Future investigation on the profit of HDRA guided treatment may provide a place for HDRA to be an adjuvant investigation for targeted or second- or third-line chemotherapies. As the biological and molecular characteristics of each cancer type differ, it is suggested that before implementation of HDRA in routine clinical practice, it should be verified whether HDRA is applicable and useful for the specific cancer type under consideration. Furthermore, one should be aware that primary culture 
is sometimes not successful due to contamination, non-growth or fibroblast overgrowth. In addition, HDRA requires a large amount of tissue which may limit the application of HDRA in clinical practice.

\section{GENOMIC PROFILING OF TUMOURS LEADS TO PERSONALIZED-PRECISION THERAPY FOR CANCER PATIENTS}

Cancer can be considered as a genome defect disease. Accumulation of genomic alterations, i.e., activation of oncogenes, suppression of tumour suppressor genes, mutation of several genes and alteration in epigenetics that transform normal cells to uncontrolled cancer cells are all possibilities. Basic and clinical cancer research have identified a number of mechanisms associated with cancer development and progression. The present advanced technology in sequencing, commonly known as nextgeneration sequencing (NGS), not only provides in-depth understanding of the genomic land-scape of cancers but also enables the identification of crucial genetic pathways that may be the molecular therapeutic targets. NGS makes it possible to have routine genomic studies of tumours in clinical practice. This allows clinicians and scientists to improve the prognosis of clinical outcomes in individuals and leads to further development of precision therapy.

The use of imatinib, the kinase inhibitor, for the treatment of chronic myelogenous leukaemias (CML) was the pioneer example for molecular driven therapy. Treatment with imatinib in CML patients who harboured BCR-ABL1 chromosomal translocation significantly improved their survival almost similar to those of general population ${ }^{18}$. Similarly, HER2-targeted therapies for patients with metastatic HER2-positive breast cancer dramatically increased a median survival to almost 5 years ${ }^{19}$ and improved the cure rates of early-stage HER2positive breast cancer by $35-50 \%{ }^{20}$. Currently, therapies targeting 11 somatic aberrations of tumours in 10 different cancer types were identified $^{21}$. Targeting the clinically validated predictive biomarkers has proven to benefit not only a specific type of tumour but also to benefit other tumours that possess similar genetic alterations. For example, the use of RAF and MEK inhibitors in cutaneous melanoma patients with BRAF V600 mutations effectively improved survivals of these patients $^{22}$. BRAF V600 mutations, however, were also evident in non-melanoma cancer patients who may obtain benefit from the same treatment. Currently, the clinical activities of these inhibitors have been identified in lung and thyroid cancers as well as in hairy cell leukaemia ${ }^{23,24}$. A common molecular subtypes of intrahepatic cholangiocarcinoma and hepatocellular carcinoma from Thai patients was demonstrated recently ${ }^{25}$, even these two cancers are clinically different with etiological and biological heterogeneity. In this context, targeted therapy should be prescribed for selected subsets of patients according to the genomic alterations of the tumour regardless of tumour type or tumour origin. Hence a new taxonomy of human cancers based on genomic profiling of abnormal mutation has been suggested to complement current histology-based classifications ${ }^{26}$.

Advances in NGS technologies have generated a large volume of data supporting somatic alterations in cancer that can be targeted for precision cancer therapy. Genomic profiling of tumours could identify the most appropriate targeted therapy to an individual patient who harbours a particular genetic profile or molecular feature. Broadening the use of targeted therapy in a larger cohort with multiple types of tumours will maximize the utilization of precision medicine in cancer. Other factors, however, may alter the responsiveness of cancer to targeted therapy and have to be taken into account. Genomic variants and the tumour microenvironment of an individual may modulate the sensitivity of the patient to a specific inhibitor of targeted therapy. These factors may signify the complexity of genomic data and decision-making for clinical implications. Finally, collaborative genome-driven clinical trials should be initiated worldwide to refine a better understanding of the roles of these genomic aberrations in association with disease pathogenesis, drug response and resistance that may lead to discovery of new drug target.

\section{DRUG REPOSITIONING AS A PROMISING STRATEGY FOR NEW ANTICANCER AGENTS}

Drug repositioning or drug repurposing for the identification of new therapeutic indications is the goal for already approved drugs. This approach is a promising strategy for drug discovery and development, as the traditional drug discovery approach is more time consuming and expensive. The repositioning is not only faster and cheaper to translate from bench to clinic but is also a benefit for patients as it reduces safety risks and accelerates successful access to treatment ${ }^{27,28}$. Drug repurposing, however, is not completely safe or ready to use as biological activity, pharmacokinetics, and clinical observations such as adverse effects, new dosing and scheduling in a new set of patients, are needed 
to be fully analysed.

Currently, there are several reports on using existing drugs as antitumor agents. For example, metformin, a standard drug for diabetes, has been reported to reduce the carcinogenic risk and inhibit tumour cell growth in endometrial, breast and ovarian cancers. The efficacy of metformin for the treatment of these cancers has been suggested in preclinical studies and clinical trials. In addition, the combination of metformin and doxorubicin, a chemotherapeutic agent, effectively inhibited growth of breast cancer stem cells and cancer cells in culture ${ }^{29}$. The treatment in a xenograft mouse model not only reduced tumour mass but also prevents relapse of cancer. The study supported the rationale of using a combination of metformin and chemo-drugs to improve treatment of breast cancer patients. In the experience of the present authors, metformin exhibited significantly anti-proliferative activity on cholangiocarcinoma cells in a dose and time dependent fashion ${ }^{30}$. Furthermore, a low dose of metformin could potentially increase anoikis and inhibit migration and invasion of cancer cells. These findings encourage the repurposing of metformin in clinical trials to improve treatment of this cancer. Several anti-parasitic drugs were also investigated for their efficacies to repurpose as anticancer agents; such as mebendazole, chloroquine, and artesunate. Anticancer activity of mebendazole as an anthelmintic drug that has been used extensively for gastro-intestinal parasitic infections in humans, has been shown effective in preclinical studies in various types of cancers, e.g., lung, brain, melanoma and cholangiocarcinoma ${ }^{31-33}$.

Recently, computational techniques and methods have been proposed for repositioning of cancer drugs. It can be 'on-target' repositioning when known pharmacological activity is applied to a different clinical application or 'off-target' repositioning, if a new mechanism has been identified for a known drug. There are different approaches to re-investigate known drugs for cancer treatment, i.e., target based, drug based and disease based ${ }^{34}$. Computational and experimental methods are needed to search for the potential repurposing drugs. Computational technologies are helpful to integrate data from various sources, i.e., pharmacologic, genomic, phenotypic, chemical and clinical information. Prediction of drug-disease responses could be obtained using bio-informatic technologies, machine learning-based models, biological network analysis and text-mining research. For validation of candidate compounds suggested by com- putational analyses, both in vitro and in vivo are necessary to be performed. Deeper understanding of genomics and molecular pathways and drug activity associated with cancer are essential to support the potential application of drug repurposing in personalized therapy.

Drug repurposing for cancer in Thailand is still young and most of research is at the preclinical level. A number of natural and synthetic compounds with anticancer activities have been reported. None, however, have been continuously validated and translated to patients. A collaborative network among investigators with different expertise including computational researchers, bio-informaticians, cell biologists and molecular biologists, as well as clinicians should be set up. Research programs, new tools and approaches for managing data should be developed from databases of Thai patients. These issues are necessary to fully realize the promise of drug repurposing in personalized medicine.

\section{Immunotherapy for cancer}

In general, immune cells can recognize cancer cells and eradicate them. Cancer cells, however, can develop a system to evade immune response and hence cancer develops. Over the past several years, immunotherapy for cancer has emerged and the most advanced and furthest in clinical implication is Chimeric Antigen Receptor (CAR) T-cell therapy. The principal of this approach is to strengthen the power of a patient's immune system to attack tumours by engineering the T-cells themselves to enhance the immune response against a specific tumour antigen. The common procedure involves, (i) genetically modifying T-cells from the patient to express a CAR on their cell membranes, (ii) expand these T-cells in vitro, and (iii) reinfuse the CAR Tcells into the patient. Second and third generation CAR-Ts have additional co-stimulatory domains that further enhance the immune response ${ }^{35}$. Until recently, CAR T-cell therapy has shown huge remission rates, largely in patients with advanced blood cancers. In 2017, two CAR T-cell therapies were approved by the Food and Drug Administration (FDA); one for the treatment of children with acute lymphoblastic leukaemia and the other for adults with advanced lymphomas. Whether CAR T-cell therapy will be effective against solid tumours, is still questionable ${ }^{36}$.

The ability to rewire an immune system to fight cancer is an alternative therapy and a new hope for cancer patients. Immunotherapy for cancer is still in its infancy in the scientific world and 
in Thailand. There are several factors that are of concern clinically; the biomarker that could be targeted by a specific CAR T-cell therapy, the adverse consequences associated with autoimmune disease, and the tumour microenvironment, all of which could interfere with treatment outcomes ${ }^{37}$.

\section{COMMENTS}

Recent advanced technologies in molecular biology, NGS and computational biology, that provide several new directions for the precise diagnosis and effective treatment of cancer are being developed. Basic and molecular research efforts in cancer biology in Thailand are solid, some of which are at the edge of translation to clinical practice. To translate the knowledge from preclinical sciences to clinical practice, new partnerships and network of scientists in various specialities, as well as people from universities, pharmaceutical companies, and others should be formed. Several national research programs aimed towards patient outcome should be developed. The Science Society of Thailand may create a stage where lead scientists in different fields can meet and develop national programs to be submitted to the government sectors that are responsible for this issue. To accelerate the translation period, many aspects have to be generated in parallel. A national biobank for cancer research, NGS service, a database for genetic profiling of cancers from Thai patients, and animal facilities for cancer research, should be developed.

Acknowledgements: This work is supported by the Thailand Research Fund and Medical Research CouncilUK (Newton Fund) to C. Pairojkul (DBG5980004). We would like to acknowledge Prof. James A. Will, University of Wisconsin for editing of the manuscript via the Faculty of Medicine Publication Clinic (ID88-60MD), Khon Kaen University, Thailand.

\section{REFERENCES}

1. Ferlay J, Soerjomataram I, Ervik M, Dikshit R, Eser S, Mathers C, et al (2013) GLOBOCAN 2012 v1.0, Cancer Incidence and Mortality Worldwide: IARC CancerBase No. 11, International Agency for Reseach on Cancer, Lyon, France, http://globocan.iarc.fr.

2. Torre LA, Siegel RL, Ward EM, Jemal A (2016) Global cancer incidence and mortality reates and trends-an update. Canc Epidemiol Biomarkers Prev 25, 16-27.

3. MNT Editorial Team (2016) Cancer: What you need to know. Medical News Today, Healthline Media UK Ltd, Brighton, UK, www.medicalnewstoday.com/ info/cancer-oncology.
4. World Bank (2016) Thailand Economic Monitor: Aging Society and Economy-June 2016, World Bank Group, Washington, DC.

5. Tolek A, Wongkham C, Proungvitaya S, Silsirivanit A, Roytrakul S, Khuntikeo N, Wongkham S (2012) Serum $\alpha_{1} \beta$-glycoprotein and afamin ratio as potential diagnostic and prognostic markers in cholangiocarcinoma. Exp Biol Med 237, 1142-9.

6. Jing CY, Fu YP, Shen HJ, Zheng SS, Lin JJ, Yi Y, et al (2017) Albumin to gamma-glutamyltransferase ratio as a prognostic indicator in intrahepatic cholangiocarcinoma after curative resection. Oncotarget 8, 13293-303.

7. Liu L, Wang W, Zhang Y, Long J, Zhang Z, Li Q, et al (2017) Declined preoperative aspartate aminotransferase to neutrophil ratio index predicts poor prognosis in patients with intrahepatic cholangiocarcinoma after hepatectomy. Canc Res Treat 50, 538-50.

8. Pattanapairoj S, Silsirivanit A, Muisuk K, Seubwai W, Cha'on U, Vaeteewoottacharn K, et al (2015) Improve discrimination power of serum markers for diagnosis of cholangiocarcinoma using data miningbased approach. Clin Biochem 48, 668-73.

9. Jinawath N, Chamgramol Y, Furukawa Y, Obama K, Tsunoda T, Sripa B, et al (2006) Comparison of gene expression profiles between Opisthorchis viverrini and non-Opisthorchis viverrini associated human intrahepatic cholangiocarcinoma. Hepatology 44, 1025-38.

10. Chan-on W, Nairismägi ML, Ong CK, Lim WK, Dima S, Pairojkul C, et al (2013) Exome sequencing identifies distinct mutational patterns in liver flukerelated and non-infection-related bile duct cancers. Nat Genet 45, 1474-8.

11. Ong CK, Subimerb C, Pairojkul C, Wongkham S, Cutcutache I, Yu W, et al (2012) Exome sequencing of liver fluke-associated cholangiocarcinoma. Nat Genet 44, 690-3.

12. Jusakul A, Cutcutache I, Yong CH, Lim JQ, Huang MN, Padmanabhan N, et al (2017) Whole-genome and epigenomic landscapes of etiologically distinct subtypes of cholangiocarcinoma. Canc Discov 7, 1116-35.

13. National Library of Medicine (2018) Genetics Home Reference, NLM, National Institutes of Health, US Department of Health and Human Services, Bethesda, MD, https://ghr.nlm.nih.gov.

14. Kubota T, Sasano N, Abe O, Nakao I, Kawamura E, Saito T, et al (1995) Potential of the histoculture drug-response assay to contribute to cancer patient survival. Clin Canc Res 1, 1537-43.

15. Furukawa T, Kubota T, Hoffman RM (1995) Clinical applications of the histoculture drug response assay. Clin Canc Res 1, 305-11.

16. Udelnow A, Schönfelder M, Würl P, Halloul Z, Meyer F, Lippert H, Mroczkowski P (2013) In vitro chemosensitivity assay guided chemotherapy is associated 
with prolonged overall survival in cancer patients. Pol J Surg 85, 340-7.

17. Yoshimasu T, Oura S, Hirai I, Tamaki T, Kokawa Y, Hata K, et al (2007) Data acquisition for the histoculture drug response assay in lung cancer. $J$ Thorac Cardiovasc Surg 133, 303-8.

18. Bower $\mathrm{H}$, Björkholm $\mathrm{M}$, Dickman PW, Höglund M, Lambert PC, Andersson TML (2016) Life expectancy of patients with chronic myeloid leukemia approaches the life expectancy of the general population. J Clin Oncol 34, 2851-7.

19. Swain SM, Baselga J, Kim SB, Ro J, Semiglazov V, Campone M, et al (2015) Pertuzumab, trastuzumab, and docetaxel in HER2-positive metastatic breast cancer. New Engl J Med 372, 724-34.

20. Piccart-Gebhart MJ, Procter M, Leyland-Jones B, Goldhirsch A, Untch M, Smith I, et al (2005) Trastuzumab after adjuvant chemotherapy in HER2positive breast cancer. New Engl J Med 353, 1659-72.

21. Hyman DM, Taylor BS, Baselga J (2017) Implementing genome-driven oncology. Cell 168, 584-99.

22. Brose MS, Cabanillas ME, Cohen EEW, Wirth LJ, Riehl T, Yue H, et al (2016) Vemurafenib in patients with $B R A F^{\mathrm{V} 600 \mathrm{E}}$-positive metastatic or unresectable papillary thyroid cancer refractory to radioactive iodine: a non-randomised, multicentre, open-label, phase 2 trial. Lancet Oncol 17, 1272-82.

23. Planchard D, Kim TM, Mazieres J, Quoix E, Riely G, Barlesi F, et al (2016) Dabrafenib in patients with $B R A F^{\mathrm{V} 600 \mathrm{E}}$-positive advanced non-smallcell lung cancer: a single-arm, multicentre, openlabel, phase 2 trial. Lancet Oncol 17, 642-50.

24. Tiacci E, Park JH, De Carolis L, Chung SS, Broccoli A, Scott S, et al (2015) Targeting mutant BRAF in relapsed or refractory hairy cell leukemia. New Engl $J$ Med 373, 1733-47.

25. Chaisaingmongkol J, Budhu A, Dang H, Rabibhadana S, Pupacdi B, Kwon SM, et al (2017) Common molecular subtypes among asian hepatocellular carcinoma and cholangiocarcinoma. Canc Cell 32, 57-70.e3.

26. Hoadley KA, Yau C, Wolf DM, Cherniack AD, Tamborero D, $\mathrm{Ng} \mathrm{S}$, et al (2014) Multiplatform analysis of 12 cancer types reveals molecular classification within and across tissues of origin. Cell 158, 929-44.

27. Langedijk J, Mantel-Teeuwisse AK, Slijkerman DS, Schutjens MH (2015) Drug repositioning and repurposing: terminology and definitions in literature. Drug Discov Today 20, 1027-34.

28. Ashburn TT, Thor KB (2004) Drug repositioning: identifying and developing new uses for existing drugs. Nat Rev Drug Discov 3, 673-83.

29. Hirsch HA, Iliopoulos D, Tsichlis PN, Struhl K (2009) Metformin selectively targets cancer stem cells, and acts together with chemotherapy to block tumor growth and prolong remission. Canc Res 69, 7507-11.

30. Saengboonmee C, Seubwai W, Cha'on U, Sawanya- wisuth K, Wongkham S, Wongkham C (2017) Metformin exerts antiproliferative and anti-metastatic effects against cholangiocarcinoma cells by targeting STAT3 and NF-kB. Anticanc Res 37, 115-23.

31. Mukhopadhyay T, Sasaki J, Ramesh R, Roth JA (2002) Mebendazole elicits a potent antitumor effect on human cancer cell lines both in vitro and in vivo. Clin Canc Res 8, 2963-9.

32. Bai RY, Staedtke V, Aprhys CM, Gallia GL, Riggins GJ (2011) Antiparasitic mebendazole shows survival benefit in 2 preclinical models of glioblastoma multiforme. Neuro Oncol 13, 974-82.

33. Sawanyawisuth K, Williamson T, Wongkham S, Riggins GJ (2014) Effect of the antiparasitic drug mebendazole on cholangiocarcinoma growth. Southeast Asian J Trop Med Publ Health 45, 1264-70.

34. Würth R, Thellung S, Bajetto A, Mazzanti M, Florio T, Barbieri F (2016) Drug-repositioning opportunities for cancer therapy: novel molecular targets for known compounds. Drug Discov Today 21, 190-9.

35. Rodríguez Fernández C (2018) A cure for cancer? How CAR-T therapy is revolutionizing oncology. Labiotech.eu Reviews, Labiotech UG, Berlin, Germany, https://labiotech.eu/car-t-therapy-cancer-review.

36. National Cancer Institute (2017) CAR T Cells: Engineering Patients' Immune Cells to Treat Their Cancers, National Cancer Institute at the National Institutes of Health, US Department of Health and Human Services, Bethesda, MD, https://www.cancer.gov/ about-cancer/treatment/research/car-t-cells.

37. Smith AJ, Oertle J, Warren D, Prato D (2016) Chimeric antigen receptor (CAR) $\mathrm{T}$ cell therapy for malignant cancers: Summary and perspective. $J$ Cell Immunother 2, 59-68. 